\title{
Crescimento vegetativo e produção de óleo essencial de hortelã-pimenta cultivada sob malhas
}

\author{
Andressa Giovannini Costa(1), Jorge Henrique Chagas(2), \\ José Eduardo Brasil Pereira Pinto(3) e Suzan Kelly Vilela Bertolucci(3)
}

(1)Universidade Federal de Goiás, Campus Jataí/Jatobá, Rodovia BR 364, Km 192, oo 3.800, CEP 75801-615 Jataí, GO. E-mail: andressagiovannini@yahoo.com.br (2)Embrapa Trigo, Rodovia BR 285, Km 294, Caixa Postal 451, CEP 99001-970 Passo Fundo, RS. E-mail: jorge.chagas@cpac.embrapa.br (3)Universidade Federal de Lavras, Campus Universitário, Caixa Postal 3037, CEP 37200-000 Lavras, MG. E-mail: jeduardo@ufla.br, suzan@dag.ufla.br

\begin{abstract}
Resumo - O objetivo deste trabalho foi avaliar o crescimento vegetativo e o teor, o rendimento e a composição química do óleo essencial de hortelã-pimenta (Mentha piperita) cultivada sob diferentes malhas, no Município de Lavras, MG. As mudas de hortelã-pimenta foram cultivadas em cinco ambientes: pleno sol, malha preta, malha aluminizada, malha azul e malha vermelha, todas com 50\% de irradiância. Utilizou-se o delineamento experimental inteiramente casualizado, com quatro repetições e cinco plantas por parcela. Foram avaliados os parâmetros: crescimento vegetativo, teor, rendimento e análise química do óleo essencial. Plantas de hortelã-pimenta cultivadas em pleno sol e sob malhas preta e vermelha produziram maior biomassa seca de folhas e maiores teores e rendimentos do óleo essencial. Entretanto, a pleno sol, o óleo essencial das plantas apresentou maior teor de mentol e, sob as malhas preta e azul, de mentofurano. Portanto, é possível manipular o crescimento das plantas e a produção e a composição química do óleo essencial de hortelã-pimenta mediante o cultivo sob malhas ou pleno sol.
\end{abstract}

Termos para indexação: Mentha piperita, mentol, mentona, qualidade da luz, radiação.

\section{Vegetative growth and yield of essential oil of peppermint grown under nets}

\begin{abstract}
The objective of this work was to evaluate the growth and the content, yield, and chemical composition of the essential oil of peppermint (Mentha piperita) grown under different nets in Lavras, MG, Brazil. Peppermint plants were grown in five environments: full sun, black net, aluminet net, blue net, and red net, all with $50 \%$ of irradiance. The experimental design was a completely randomized block with four replicates and five plants per plot. The evaluated parameters were: vegetative growth, content, yield, and chemical analysis of the essential oil. Peppermint plants grown under full sun and black and red nets produced higher dry leaf biomass and higher essential oil content and yield. However, in full sun, the essential oil of the plants had higher contents of menthol and, under black and blue nets, of menthophurane. Therefore, it is possible to manipulate plant growth and yield and chemical composition of the essential oil of peppermint with cultivation under nets or full sun.
\end{abstract}

Index terms: Mentha piperita, menthol, menthone, light quality, radiation.

\section{Introdução}

A hortelã-pimenta (Mentha piperita L.) está entre os mais populares ingredientes de chás. As ações medicinais desta planta agem sobre desordens biliares, dispepsia, enterite, flatulência e espasmos intestinais (McKay \& Blumberg, 2006). A espécie também é fonte de um dos mais populares óleos essenciais, com diversas aplicações nas indústrias de alimentos, cosmética e farmacêutica. Os mais importantes constituintes do óleo de hortelã-pimenta são: mentol, mentona, mentofurano, acetato de mentila e pulegona (Aflatuni, 2005). A composição qualitativa e quantitativa desses monoterpenoides determina a qualidade e o valor comercial do óleo essencial. O óleo de menta de alta qualidade consiste, predominantemente, de mentol
(30-55\%); seguido de moderada proporção de seu precursor, mentona (14-32\%); e de baixos teores de pulegona $(<4 \%)$, mentofurano $(1-9 \%)$ e acetato de mentila (2,8-10\%) (Behn et al., 2010). Entretanto, os teores dos constituintes químicos são variáveis, conforme a localização geográfica do cultivo ou o resultado da combinação de diversos outros fatores, tais como genótipo, ontogenia, luz, temperatura, água e nutrientes (Aflatuni, 2005).

A luz é o fator preponderante no controle do crescimento, do desenvolvimento e do metabolismo das plantas, os quais são afetados por sua intensidade, direção, duração e qualidade (Almeida \& Mundstock, 2001; Chang et al., 2008).

A grande variação do clima e as exigências do mercado têm feito com que muitos produtores 
procurem novas tecnologias de produção. Uma das novidades no cultivo protegido é a introdução de filtros espectrais, os quais transmitem seletivamente certos comprimentos de ondas e, consequentemente, alteram a resposta fotomorfogênica das plantas. Essas respostas se traduzem em alterações no crescimento, no desenvolvimento, na morfologia e nas funções fisiológicas das plantas, como resultado à adaptação a uma condição ambiental diferente (Tsormpatsidis et al., 2008).

A malha vermelha reduz as ondas azuis, verdes e amarelas, e acrescenta ondas na faixa espectral do vermelho e do vermelho distante, com transmitância para comprimentos de ondas superiores a $590 \mathrm{~nm}$. Já a malha azul, filtra as ondas na faixa do vermelho e do vermelho distante, o que permite a passagem de ondas com transmitância na região do azul-verde (400-540 nm) (Shahak et al., 2004). A malha preta (sombrite) é considerada neutra e auxilia apenas na redução da incidência de radiação sobre as plantas, sem influência na qualidade espectral da luz (Oren-Shamir et al., 2001).

A luz vermelha atua no desenvolvimento da estrutura fotossintética das plantas, o que pode aumentar o acúmulo de amido em algumas espécies, pela inibição da translocação de assimilados para fora das folhas (Saebo \& Mortensen, 1996). A luz azul permite alterar o crescimento, o desenvolvimento e a aclimatação às condições ambientais das plantas (Taiz \& Zeiger, 2004). Assim, a utilização de malhas com transmissão seletiva de radiação UV pode oferecer um caminho para aumentar a produção de metabólitos secundários em plantas medicinais e aromáticas. Contudo, há poucos estudos sobre os efeitos da radiação UV no crescimento, no desenvolvimento e na produção de metabólitos das plantas (Tsormpatsidis et al., 2008).

Outra nova geração de malha é a termorrefletora, que tem sido recentemente adotada no Brasil. Essas malhas permitem manejar a diferença de temperatura entre o dia e a noite, o que possibilita a formação de diversos microclimas, de acordo com a malha utilizada, e, também, protegem as plantas da radiação solar excessiva e conservam o calor no interior do ambiente (Leite et al., 2008). No entanto, o cultivo protegido tradicional, sem o uso de malhas coloridas, além de exigir estrutura onerosa, proporciona efeito estufa pela cobertura de plástico utilizada, que é prejudicial em épocas quentes do ano. As telas de sombreamento (sombrites), na maioria das vezes, não proporcionam níveis adequados de luz, o que afeta o desenvolvimento e a produção das plantas (Corrêa, 2008).

Em plantas medicinais, diversos trabalhos têm mostrado a influência das malhas na produção de biomassa e nos teores de metabólitos secundários sob condições ambientais controladas, por induzirem alterações fisiológicas e bioquímicas na planta (Abreu \& Mazzafera, 2005; Afreen et al., 2005; Pinto et al., 2007; Corrêa, 2008; Costa et al., 2010).

O objetivo deste trabalho foi avaliar o crescimento vegetativo e o teor, o rendimento e a composição química do óleo essencial de hortelã-pimenta cultivada sob diferentes malhas, no Município de Lavras, MG.

\section{Material e Métodos}

O experimento foi conduzido entre março e julho de 2009 , na Fazenda Gota de Esperança $\left(21^{\circ} 14^{\prime} 07^{\prime \prime} \mathrm{S}\right.$ e $44^{\circ} 58^{\prime} 22^{\prime \prime W}$, a 879 m de altitude), do Departamento de Agricultura (DAG), da Universidade Federal de Lavras (Ufla). As análises de crescimento e a extração de óleo essencial foram feitas no Laboratório de Cultura de Tecidos e Plantas Medicinais da Ufla. De acordo com Köppen, o clima da região é do tipo Cwa, mas apresenta características de Cwb, com duas estações bem definidas, uma fria e seca, de abril a setembro, e outra quente e úmida, de outubro a março (Brasil, 1992). Durante o experimento, foram observadas temperaturas médias mensais de $20,9^{\circ} \mathrm{C}$, com mínima de $13^{\circ} \mathrm{C}$ e máxima de $31,6^{\circ} \mathrm{C}$, e precipitação média mensal de 136,26 mm. Conforme dados coletados na Estação Climatológica Principal de Lavras, da Ufla, a umidade relativa média do ar foi de $74 \%$, tendo variado entre 66 e $80 \%$, e a insolação média foi de 6,3 horas, tendo variado de 4,0 a 7,5 horas.

As mudas foram obtidas a partir de miniestacas apicais de aproximadamente $5,0 \mathrm{~cm}$ de comprimento, coletadas de plantas matrizes do Horto de Plantas Medicinais do DAG, da Ufla, enraizadas em bandejas de poliestireno expandido de 128 células, tendo-se utilizado substrato comercial (Plantmax). Após o enraizamento, as mudas foram transplantadas para vasos de plástico de $10 \mathrm{~L}$ de capacidade, contendo uma mistura de terra, areia e esterco bovino (3:1:1). O solo utilizado como substrato foi coletado na camada de $0-20 \mathrm{~cm}$ de profundidade, de um Latossolo Vermelho-Amarelo distrófico (Santos et al., 2006), em Lavras, MG. Foram realizadas análises do solo e do substrato no Laboratório de Análises Química e Física do Solo, do Departamento de Ciência do Solo, da Ufla. 
Utilizou-se o delineamento experimental inteiramente casualizado, com cinco tratamentos e quatro repetições. Cada parcela foi constituída por cinco plantas (uma planta por vaso). As plantas foram cultivadas em telados com uma área de 7,85x7,35 m e altura de $2,10 \mathrm{~m}$. Os tratamentos utilizados foram: malha preta (Polysack); malha termorrefletora aluminizada (Aluminet); malha azul (ChromatiNet Azul); malha vermelha (ChromatiNet Vermelha); e a pleno sol. Todas as malhas avaliadas interceptaram a radiação solar em $50 \%$.

A colheita das plantas foi realizada após 120 dias de cultivo. As plantas foram individualmente separadas em raiz, caule e folha para secagem em estufa com ventilação forçada a $40^{\circ} \mathrm{C}$ até peso constante. Para a preservação da integridade da massa das raízes, estas foram colocadas em recipientes individuais e lavadas em água corrente até total remoção do substrato. A água residual do recipiente passou por uma peneira para retenção das raízes, para posterior secagem. A massa do material vegetal seco foi obtida para determinação da biomassa seca de folhas, de caule, de raízes, da parte aérea e total da planta (g por planta), tendo-se determinado, também, a relação raiz:parte aérea. Após a colheita, com o material vegetal ainda fresco, avaliou-se a área foliar $\left(\mathrm{cm}^{2}\right.$ por planta), medida com auxílio de medidor eletrônico de área foliar, modelo LI-3100 (LICOR, Lincoln, Nebraska, EUA), a partir da qual foram obtidos os parâmetros fisiológicos de razão de área foliar $\left(\mathrm{cm}^{2} \mathrm{~g}^{-1}\right)$, área foliar específica $\left(\mathrm{cm}^{2} \mathrm{~g}^{-1}\right)$, razão de massa foliar $\left(\mathrm{g} \mathrm{g}^{-1}\right)$ e massa específica foliar (mg cm${ }^{-2}$ por planta), conforme Benincasa (2003).

O óleo essencial foi extraído de $20 \mathrm{~g}$ de folhas secas; porém, para a malha azul, foram utilizados $18 \mathrm{~g}$ de folhas secas. A extração foi realizada pelo processo de hidrodestilação, em aparelho de Clevenger modificado (Vidrolab, São Paulo, SP), por 120 min. O óleo foi separado por decantação por $5 \mathrm{~min}$, e o sobrenadante foi recolhido. Foram determinados o teor $\left(\mu \mathrm{L} 100 \mathrm{~g}^{-1}\right.$ de biomassa seca de folhas), o rendimento ( $\mathrm{mL}$ por planta) e a composição química do óleo essencial.

As análises da composição química foram realizadas por meio de amostra composta de alíquotas equivolumétricas do óleo essencial das repetições de cada tratamento, no Laboratório de Fitoquímica do DAG, da Ufla.

As análises quantitativas do óleo foram realizadas por cromatografia em fase gasosa acoplada a detector de ionização de chama de hidrogênio (CG-DIC), com uso do cromatógrafo Agilent 7890A (Agilent Technologies, Inc., Santa Clara, Califórnia, EUA), equipado com coluna capilar de sílica fundida HP-5 ( $30 \mathrm{~m}$ de comprimento $\mathrm{x} 0,25 \mathrm{~mm}$ de diâmetro interno $\mathrm{x}$ $0,25 \mu \mathrm{m}$ de espessura do filme). O gás hélio foi utilizado

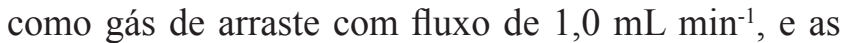
temperaturas do injetor e do detector foram mantidas em 220 e $240^{\circ} \mathrm{C}$, respectivamente. A temperatura inicial do forno foi de $60^{\circ} \mathrm{C}$, isotérmico por $1,5 \mathrm{~min}$, seguido por rampa de temperatura de $3^{\circ} \mathrm{C}$ por min até $240^{\circ} \mathrm{C}$ e rampa de $10^{\circ} \mathrm{C}$ por min até $270^{\circ} \mathrm{C}$. O óleo foi diluído em acetato de etila $\left(1 \%, \mathrm{v} \mathrm{v}^{-1}\right)$ e injetado automaticamente no cromatógrafo com uso de volume de injeção de $1,0 \mu \mathrm{L}$, no modo split, a uma razão de injeção de 1:50. A análise quantitativa foi obtida por meio da integração do cromatograma total de íons, e o teor dos constituintes eluídos foi expresso como percentagem de área relativa das áreas dos picos.

As análises qualitativas do óleo foram realizadas por cromatografia gasosa acoplada à espectrometria de massas (CG-EM), com uso de equipamento Agilent 5975C (Agilent Technologies, Inc., Santa Clara, Califórnia, EUA) operado por ionização de impacto eletrônico a $70 \mathrm{eV}$, em modo varredura, à velocidade de 1,0 scan s$^{-1}$, com intervalo de aquisição de massas de $40-400 \mathrm{~m} / \mathrm{z}$. As condições cromatográficas foram as mesmas utilizadas nas análises quantitativas.

Para identificar os componentes, os índices de retenção calculados $\left(\mathrm{IK}_{\mathrm{c}}\right)$ foram comparados aos dados de espectros de massas e de índices de retenção (IK) da literatura (Davies, 1990; Adams, 2007); e os espectros de massas foram comparados ao banco de dados da biblioteca NIST/EPA/NHI (National Institute of Standards and Technology, 2008). Os índices de retenção de Kovats $\left(\mathrm{IK}_{\mathrm{c}}\right)$ relativos à coinjeção de mistura padrão de n-alcanos, $\mathrm{C}_{8}-\mathrm{C}_{20}$ (Sigma Chemical Co., St. Louis, MO, EUA), foram calculados com a aplicação da equação de Van Den Dool \& Kratz (1963).

Para as análises estatísticas dos dados, utilizou-se o programa Sisvar, versão 5.0 (Ferreira, 2008). As médias entre os tratamentos foram submetidas à análise de variância, pelo teste $\mathrm{F}$, e foram comparadas pelo teste de Scott-Knott, a 5\% de probabilidade.

\section{Resultados e Discussão}

A biomassa total das plantas cultivadas a pleno sol e sob malha vermelha foi maior que a das demais, e o 
menor acúmulo ocorreu nas plantas cultivadas sob malha azul (Tabela 1). Afreen et al. (2005) e Shahak (2008) observaram maior crescimento de plantas de Glycyrrhiza uralensis Fisch. ex DC., Lactuca sativa L. e Ocimum basilicum L. sob malha vermelha do que sob malha azul, sombrite e termorrefletora. De acordo com Shahak (2008), o maior acúmulo de biomassa por plantas cultivadas sob malha vermelha, em comparação às cultivadas sob malha azul, pode ser atribuído ao fato de a malha vermelha estimular a taxa de crescimento e o vigor vegetativo, enquanto a malha azul impede o crescimento e o desenvolvimento da planta.

As biomassas secas de folhas, de caule e da parte aérea foram significativamente superiores nos cultivos sob pleno sol, malha vermelha e malha preta, quando comparadasàs dos demaisambientes decultivo. Entretanto, apesar de as plantas de hortelã-pimenta cultivadas sob malha preta terem apresentado ganho na parte aérea, o mesmo não foi observado para o crescimento das raízes em plantas cultivadas sob pleno sol e malha vermelha. Ao se avaliar a relação raiz:parte aérea, os cultivos sob malha preta e azul foram os que apresentaram menor crescimento das raízes em valor numérico $(0,20$ e 0,21 , respectivamente) (Tabela 1). Afreen et al. (2005) também observaram redução significativa no crescimento das raízes de plantas de $G$. uralensis sob incidência de luz azul. De modo geral, o desenvolvimento das plantas de hortelã-pimenta foi afetado de forma negativa quando cultivadas sob malha azul.

As biomassas secas de raiz de hortelã-pimenta sob as malhas vermelha e termorrefletora não diferiram entre si, porém, foram menores em comparação à biomassa seca de raiz de plantas cultivadas a pleno sol. A malha termorrefletora diminui a temperatura

Tabela 1. Acúmulo de biomassa seca em plantas de Mentha piperita cultivadas sob diferentes malhas ${ }^{(1)}$.

\begin{tabular}{|c|c|c|c|c|c|c|}
\hline \multirow[t]{2}{*}{ Tratamento } & BSF & BSC & BSR & BPA & BST & \multirow[t]{2}{*}{$\mathrm{R} / \mathrm{PA}$} \\
\hline & \multicolumn{5}{|c|}{--------------------- $\left(\right.$ g planta'1 $\left.^{-1}\right)$------------------- } & \\
\hline Pleno sol & $6,71 \mathrm{a}$ & $15,99 \mathrm{a}$ & $22,56 a$ & $22,20 \mathrm{a}$ & $45,26 a$ & $0,047 \mathrm{a}$ \\
\hline Malha termorrefletora & $4,19 b$ & $10,47 b$ & $12,99 \mathrm{~b}$ & $15,64 b$ & $27,66 b$ & $0,031 b$ \\
\hline Malha preta & $7,06 \mathrm{a}$ & $15,00 \mathrm{a}$ & $2,91 \mathrm{c}$ & $18,72 \mathrm{a}$ & $24,97 b$ & $0,020 \mathrm{c}$ \\
\hline Malha azul & $3,79 b$ & $9,92 b$ & $6,09 \mathrm{c}$ & $11,70 \mathrm{~b}$ & $19,81 \mathrm{c}$ & $0,021 \mathrm{c}$ \\
\hline Malha vermelha & $6,88 \mathrm{a}$ & $17,37 \mathrm{a}$ & $15,55 \mathrm{~b}$ & $21,14 \mathrm{a}$ & $39,81 \mathrm{a}$ & $0,031 b$ \\
\hline Média & 5,73 & 13,75 & 19,89 & 17,88 & 31,50 & 0,030 \\
\hline CV (\%) & 16,51 & 15,15 & 12,02 & 21,97 & 12,88 & 23,36 \\
\hline
\end{tabular}

${ }^{(1)}$ Médias seguidas de letras iguais, nas colunas, não diferem pelo teste de Scott-Knott, a 5\% de probabilidade. BSF, biomassa seca de folhas; BSC, biomassa seca do caule; BSR, biomassa seca da raiz; BPA, biomassa seca da parte aérea; BST, biomassa total; e R/PA, relação raiz: parte aérea. ambiente e reduz as temperaturas do substrato e da planta, o que melhora as condições de absorção de nutrientes e o desenvolvimento radicular no substrato. Na planta, proporciona maior condutância estomática, o que facilita a absorção de carbono. Contudo, não foi observado incremento na biomassa de raiz e na parte aérea das plantas cultivadas sob malha termorrefletora, em comparação às plantas a pleno sol. Chagas et al. (2010) caracterizaram a hortelã-japonesa (M. arvensis), espécie do mesmo gênero da hortelã-pimenta, como sendo uma "planta de sol", na qual a radiação solar plena proporciona ganhos significativos em sua biomassa.

O ganho de biomassa seca de folhas nas plantas de hortelã-pimenta cultivadas sob as malhas preta e vermelha (Tabela 1) pode estar relacionado ao incremento da área foliar $\left(1.276,13\right.$ e $1.343,62 \mathrm{~cm}^{2}$ por planta, respectivamente) (Tabela 2). Resultados similares foram observados em hortelã-japonesa cultivada sob diferentes malhas por Chagas et al. (2010), que atribuíram o aumento da área foliar nas plantas mais à intensidade do que à qualidade espectral da luz. De acordo com Taiz \& Zeiger (2004), como estratégia adaptativa, as plantas submetidas a baixos níveis de irradiância expandem as folhas para aumentar a captação da energia luminosa e permitir maior eficiência fotossintética e, consequentemente, maior fixação de carbono. Ao contrário do observado por Chagas et al. (2010), no presente trabalho, a expansão foliar, em hortelã-pimenta, parece estar relacionada mais à qualidade do que à intensidade da luz, conforme observado em plantas cultivadas sob diferentes malhas (vermelha e preta) com a mesma intensidade de radiação $(50 \%)$.

Tabela 2. Crescimento em plantas de Mentha piperita cultivadas sob diferentes malhas ${ }^{(1)}$.

\begin{tabular}{lccccc}
\hline Tratamento & $\begin{array}{c}\text { AF } \\
\left(\mathrm{cm}^{2} \mathrm{planta}^{-1}\right)\end{array}$ & $\begin{array}{c}\text { RPF } \\
\left(\mathrm{g} \mathrm{g}^{-1}\right)\end{array}$ & $\begin{array}{c}\text { RAF } \\
------\end{array}$ & $\begin{array}{c}\text { AFE } \\
\left(\mathrm{cm}^{2} \mathrm{~g}^{-1}\right)----\end{array}$ & $\begin{array}{c}\text { PEF } \\
\left(\mathrm{mg} \mathrm{cm}^{-2} \mathrm{planta}^{-1}\right)\end{array}$ \\
\hline Pleno sol & $971,29 \mathrm{~b}$ & $0,150 \mathrm{~b}$ & $21,72 \mathrm{c}$ & $149,12 \mathrm{a}$ & $6,70 \mathrm{a}$ \\
Malha termorrefletora & $923,40 \mathrm{~b}$ & $0,152 \mathrm{~b}$ & $33,20 \mathrm{~b}$ & $230,34 \mathrm{a}$ & $4,89 \mathrm{a}$ \\
Malha preta & $1.276,13 \mathrm{a}$ & $0,282 \mathrm{a}$ & $51,59 \mathrm{a}$ & $182,33 \mathrm{a}$ & $5,60 \mathrm{a}$ \\
Malha azul & $935,19 \mathrm{~b}$ & $0,190 \mathrm{~b}$ & $47,62 \mathrm{a}$ & $275,34 \mathrm{a}$ & $4,13 \mathrm{a}$ \\
Malha vermelha & $1.343,62 \mathrm{a}$ & $0,175 \mathrm{~b}$ & $35,22 \mathrm{~b}$ & $200,55 \mathrm{a}$ & $5,31 \mathrm{a}$ \\
\hline Média & $1.089,92$ & 0,19 & 37,87 & 207,54 & 5,38 \\
CV $(\%)$ & 15,91 & 16,22 & 21,43 & 36,76 & 28,68 \\
\hline
\end{tabular}

${ }^{(1)}$ Médias seguidas de letras iguais, nas colunas, não diferem pelo teste de Scott-Knott, a 5\% de probabilidade. AF, área foliar; RPF, razão de massa foliar; RAF, razão de área foliar; AFE, área foliar específica; e PEF, massa específica foliar. 
Os aumentos na razão de área foliar constituem uma adaptação da planta à baixa luminosidade, o que indica maior proporção de tecido fotossinteticamente ativo na forma de área foliar (Benincasa, 2003). A menor razão de área foliar foi observada nas plantas cultivadas a pleno sol e aumentou nas malhas termorrefletora e vermelha, tendo atingido o máximo nas malhas preta e azul (Tabela 2). Esse resultado é indicativo de que, sob as malhas azul e preta, o crescimento de hortelã-pimenta é comprometido, pois as plantas requereram maior área foliar para a produção de um grama de matéria seca, em comparação às demais.

Ao se considerar que as folhas são o centro de produção de biomassa e que o resto da planta depende da exportação de material da folha, a razão do peso foliar expressa a fração de biomassa não exportada das folhas para outras partes da planta (Benincasa, 2003). A malha preta apresentou maior razão de massa foliar e menor translocação de material das folhas para as demais partes da planta, quando comparada aos outros tratamentos, o que explica o baixo acúmulo de biomassa seca de raiz (Tabela 2).

Esses resultados mostram diferentes respostas de crescimento de plantas de hortelã-pimenta em relação às diferentes condições de qualidade espectral da luz fornecidas pelo uso de malhas coloridas. Assim, o presente trabalho corrobora o comportamento de plantas de hortelã-pimenta frente às novas tecnologias de produção de vegetais, o que indica que o cultivo sob as malhas afeta o crescimento da espécie.

Quanto ao teor e ao rendimento do óleo essencial de hortelã-pimenta, houve diferenças significativas entre as plantas cultivadas nos diferentes ambientes de produção (Tabela 3). Os maiores valores foram observados nas plantas cultivadas a pleno sol e sob as malhas preta e vermelha, em comparação aos demais tratamentos. Na literatura, há relatos do aumento do teor de óleo essencial em plantas aromáticas com o aumento da taxa de luminosidade (Burbott \& Loomis,
1967; Chang et al., 2008; Costa et al., 2010); contudo, no presente trabalho, isso não ficou evidenciado, pois esse aumento foi observado tanto no cultivo a pleno sol quanto no cultivo sob malhas, o que parece estar mais relacionado a algum tipo de resposta associada à qualidade da luz incidente do que à intensidade luminosa.

Os teores de óleos essenciais de hortelã-pimenta encontrados na literatura variam entre 0,9 e $3,9 \%\left(\mathrm{v} \mathrm{p}^{-1}\right)$ (McKay \& Blumberg, 2006), e o teor médio observado no presente trabalho está situado nessa faixa (Tabela 3).

A análise química do óleo essencial de hortelã-pimenta identificou sete monoterpenos que constituem 93\% da composição química total do óleo essencial. Observouse que a qualidade da luz afetou a composição quantitativa dos monoterpenos, particularmente nos teores dos constituintes majoritários, representados por mentona, mentofurano, mentol e acetato de mentila (Tabela 4). Limoneno, pulegona e acetato de neomentila também foram identificados no óleo de hortelã-pimenta.

O maior teor de mentol foi observado sob cultivo a pleno sol. Estudos sobre a influência da radiação UV-B e a luz fotossinteticamente ativa no teor de monoterpenos do óleo essencial de folhas de hortelã-pimenta foram

Tabela 3. Teor e rendimento de óleo essencial de Mentha piperita cultivada sob diferentes malhas ${ }^{(1)}$.

\begin{tabular}{lcc}
\hline Tratamento & Teor $\left(\mu \mathrm{L} 100 \mathrm{~g}^{-1}\right)$ & Rendimento $\left(\mathrm{mL}\right.$ planta $\left.^{-1}\right)$ \\
\hline Pleno sol & $1,302 \mathrm{a}$ & $0,088 \mathrm{a}$ \\
Malha termorrefletora & $0,967 \mathrm{~b}$ & $0,041 \mathrm{~b}$ \\
Malha preta & $1,332 \mathrm{a}$ & $0,093 \mathrm{a}$ \\
Malha azul & $0,997 \mathrm{~b}$ & $0,037 \mathrm{~b}$ \\
Malha vermelha & $1,067 \mathrm{a}$ & $0,073 \mathrm{a}$ \\
\hline Média & 1,133 & 0,066 \\
$\mathrm{CV}(\%)$ & 16,16 & 22,70 \\
\hline
\end{tabular}

${ }^{(1)}$ Médias seguidas de letras iguais, nas colunas, não diferem pelo teste de Scott-Knott, a 5\% de probabilidade.

Tabela 4. Percentagens relativas dos compostos presentes no óleo essencial de Mentha piperita cultivada sob diferentes malhas.

\begin{tabular}{lcccccccc}
\hline Tratamento & Limoneno & Mentona & Mentofurano & Mentol & Pulegona & Acetato de neomentila & Acetato de mentila & Total \\
\hline $\mathrm{IK}^{(1)}$ & 1.012 & 1.142 & 1.153 & 1.163 & 1.237 & 1.278 & - & 1.299 \\
Pleno sol & 2,82 & 10,42 & 20,62 & 20,96 & 1,95 & 2,37 & 32,98 & 92,12 \\
Malha termorrefletora & 2,78 & 7,47 & 29,21 & 15,86 & 2,55 & 2,79 & 31,68 & 92,34 \\
Malha preta & 3,06 & 5,63 & 38,75 & 13,99 & 2,53 & 1,96 & 27,75 & 93,67 \\
Malha azul & 2,49 & 5,48 & 34,99 & 9,43 & 3,67 & 2,85 & 35,84 & 94,75 \\
Malha vermelha & 2,78 & 3,80 & 27,77 & 11,10 & 1,44 & 3,52 & 41,89 & 92,30 \\
\hline
\end{tabular}

${ }^{(1)} \mathrm{IK}$, índice de Kovats calculado em relação à retenção de n alcanos (C8 a C20) em coluna HP-5. 
realizados por Behn et al. (2010), o que confirma a maior produção de mentol sob pleno sol. O uso das malhas parece ocasionar redução no teor de mentol, que foi de cerca da metade do observado a pleno sol nas malhas azul e vermelha. Maffei et al. (1999) também verificaram redução na biossíntese de mentol em plantas de hortelã-pimenta sob exposição à luz azul. $\mathrm{O}$ teor de mentona apresentou maior biossíntese nas plantas cultivadas a pleno sol e menor produção no cultivo sob malhas. Para o mentofurano, o cultivo a pleno sol proporcionou reduções acentuadas, enquanto nas malhas, foram observados os melhores resultados (Tabela 4).

Resultadossimilares foram obtidos em outros estudos. Segundo Maffei et al. (1999), a composição química do óleo de hortelã-pimenta é fortemente influenciada por fatores ambientais. Estes autores observaram redução de $65 \%$ no teor de mentol na luz azul e aumento de cerca de 2,3 vezes no teor de mentofurano, em comparação à luz branca. Aflatuni (2005) relatou que plantas de hortelã-pimenta submetidas a dias curtos apresentaram quantidades muito pequenas de mentol e mentona, além de altos teores de mentofurano.

A relação da qualidade da luz e a composição do óleo essencial de hortelã-pimenta pode estar associada à rota de biossíntese dos compostos. $\mathrm{Na}$ síntese do óleo essencial de hortelã-pimenta, a (+)-pulegona é a precursora do mentol, considerado o componente mais significativo dos óleos de Mentha spp. Dependendo das condições ambientais, a (+)-pulegona pode reduzir a (-)-mentona e sintetizar o mentol, por meio da pulegona redutase, ou oxidar o (+)-mentofurano, pela enzima mentofurano sintase (Mahmoud \& Croteau, 2003). Conforme Burbott \& Loomis (1967), o nível de oxidação-redução dos monoterpenos reflete no estado de oxidação-redução das coenzimas respiratórias de síntese dos terpenos, o que depende da concentração do substrato respiratório nas células. Com base nesta suposição, a radiação solar possivelmente preserva altos níveis de substratos respiratórios e mantém as condições redutivas, enquanto, sob as radiações proporcionadas pelas malhas, há perda dos substratos respiratórios, o que resulta em condições oxidativas.

A farmacopeia europeia estabelece que o óleo de hortelã-pimenta deve conter teor de $30-55 \%$ de mentol, $14-32 \%$ de mentona, $2,8-10 \%$ de acetato de mentila e 1-9\% de mentofurano (Harris, 2006). Independentemente da qualidade de luz imposta à hortelã-pimenta cultivada em Lavras, MG, os teores de acetato de mentila e mentofurano são elevados e não preenchem a esses requisitos. Porém, essa composição química pode ser interessante nas formulações de perfumes, por não intensificar o aroma da essência, além de ser uma substância cara, em virtude da limitada disponibilidade e da baixa produção pelas plantas (0 a 6\%). Deve-se considerar, também, a aceitabilidade do mentofurano sintético, na indústria dos aromas, como fator determinante no custo de óleo essencial que contenha componentes sintéticos (Khanuja et al., 2003).

\section{Conclusões}

1. É possível manipular o cultivo de Mentha piperita, bem como a produção e a composição química do óleo essencial com o uso de malhas ou o cultivo a pleno sol.

2. Plantas de $M$. piperita cultivadas sob pleno sol e malhas preta e vermelha produzem maior biomassa seca de folhas e maiores teores e rendimentos do óleo essencial.

3. Os óleos essenciais das plantas cultivadas a pleno sol apresentam maior teor de mentol, enquanto os das sob as malhas preta e azul apresentam maiores teores de mentofurano.

\section{Referências}

ABREU, I.N. de; MAZZAFERA, P. Effect of water and temperature stress on the content of active constituents of Hypericum brasiliense Choisy. Plant Physiology and Biochemistry, v.43, p.241-248, 2005.

ADAMS, R.P. Identification of essential oil components by gas chromatography/mass spectrometry. $4^{\text {th }}$ ed. Illinois: Allured Publishing Corporation, 2007. 804p.

AFLATUNI, A. The yield and essential oil content of mint (Mentha ssp.) in northern ostrobothnia. 2005. 50p. Dissertation (Academic) - University of Oulu, Oulu.

AFREEN, F.; ZOBAYED, S.M.A.; KOZAI, T. Spectral quality and UV-B stress stimulate glycyrrhizin concentration of Glycyrrhiza uralensis in hydroponic and pot system. Plant Physiology and Biochemistry, v.43, p.1074-81, 2005.

ALMEIDA, M.L. de; MUNDSTOCK, C.M. O afilhamento da aveia afetado pela qualidade da luz em plantas sob competição. Ciência Rural, v.31, p.393-400, 2001.

BEHN, H.; ALBERT, A.; MARX, F.; NOGA, G.; ULBRICH, A. Ultraviolet-B and photosynthetically active radiation interactively affect yield and pattern of monoterpenes in leaves of peppermint (Mentha x piperita L.). Journal of Agricultural and Food Chemistry, v.58, p.7361-7367, 2010. 
BENINCASA, M.M.P. Análise de crescimento de plantas. Jaboticabal: FUNEP, 2003. 41p.

BRASIL. Ministério da Agricultura e Reforma Agrária. Normais climatológicas de 1961 - 1990. Brasília: Ministério da Agricultura e Reforma Agrária, 1992. 84p.

BURBOTT, A.J.; LOOMIS, W.D. Effects of light and temperature on the monoterpenes of peppermint. Plant Physiology, v.42, p.20-28, 1967.

CHAGAS, J.H.; PINTO, J.E.B.P.; BERTOLUCCI, S.K.V.; FERRAZ, E. de O.; BOTREL, P.P.; SANTOS, F.M. dos. Produção de biomassa seca em plantas de Mentha arvensis L. cultivada sob malhas fotoconversoras. Horticultura Brasileira, v.28, p.3422-3427, 2010.

CHANG, X.; ALDERSON, P.G.; WRIGHT, C.J. Solar irradiance level alters the growth of basil (Ocimum basilicum L.) and its content of volatile oils. Environmental and Experimental Botany, v.63, p.216-223, 2008.

CORRÊA, R.M. Adubação orgânica, intensidade e qualidade de luz no crescimento de plantas, características anatômicas e composição química do óleo essencial de orégano (Origanum vulgare L.). 2008. 131p. Tese (Doutorado) - Universidade Federal de Lavras, Lavras.

COSTA, L.C. do B.; PINTO, J.E.B.P.; CASTRO, E.M.; ALVES, E.; ROSAL, L.F.; BERTOLUCCI, S.K.V.; ALVES, P.B.; EVANGELINO, T.S. Yield and composition of the essential oil of Ocimum selloi Benth. cultivated under colored netting. Journal of Essential Oil Research, v.22, p.34-39, 2010.

DAVIES, N.W. Gas chromatographic retention indices of monoterpenes and sesquiterpenes on methyl silicone and Carbowax 20M phases. Journal of Chromatography, v.503, p.1-24, 1990.

FERREIRA, D.F. SISVAR: um programa para análises e ensino de estatística. Revista Symposium, v.6, p.36-41, 2008.

HARRIS, B. Menthol: a review of its thermoreceptor interactions and their therapeutic applications. International Journal of Aromatherapy, v.16, p.117-131, 2006.

KHANUJA, S.P.S.; SINGH, A.K.; BAHAL, J.R.; DAROKAR, M.P.; GUPTA, S.; KUMAR, B.; LAL, R.K.; PATRA, N.K.; PRIYA, T.P.; SHASANY, A.; TOMAR, V.K.S.; UPADHYAY, R.K. Mint plant named 'Cim Indus'. PP16474 n. 10749906, 29 Dec. 2003. Available at: <http:// www.patentstorm.us/patents/PP16474/ description.html>. Accessed on: 06 May 2011.

LEITE, C.A.; ITO, R.M.; LEE, G.T.S.; GANELEVIN, R.; FAGNANI, M.A. Light spectrum management using colored nets to control the growth and blooming of phalaenopsis. Acta Horticulturae, v.770, p.177-184, 2008.

MAFFEI, M.; CANOVA, D.; BERTEA, C.M.; SCANNERINI, S. UV-A effects on photomorphogenesis and essential oil composition in Mentha piperita. Journal of Photochemistry and Photobiology B: Biology, v.52, p.105-110, 1999.
MAHMOUD, S.S.; CROTEAU, R.B. Menthofuran regulates essential oil biosynthesis in peppermint by controlling a downstream monoterpene reductase. Proceedings of the National Academy of Sciences of the United States of America, v.100, p.14481-14486, 2003.

MCKAY, D.L.; BLUMBERG, J.B. A review of the bioactivity and potential health benefits of peppermint tea (Mentha piperita L.). Phytotherapy Research, v.20, p.619-633, 2006.

NATIONALINSTITUTEOFSTANDARDSANDTECHNOLOGY. PC version 2.0 of the NIST/EPA/NIH Mass Spectral Library. Gaithersburg: NIST, 2008.

OREN-SHAMIR, M.; GUSSAKOVSKY, E.E.; SHPIEGEL, E.; NISSIM-LEVI, A.; RATNER, K.; OVADIA, R.; GILLER, Y.E; SHAHAK, Y. Coloured shade nets can improve the yield and quality of green decorative branches of Pittosphorum variegatum. Journal of Horticultural Science and Biotechnology, v.76, p.353-361, 2001.

PINTO, J.E.B.P.; CARDOSO, J.C.W.; CASTRO, E.M. de; BERTOLUCCI, S.K.V.; MELO, L.A. de; DOUSSEAU, S. Aspectos morfofisiológicos e conteúdo de óleo essencial de plantas de alfazema-do-Brasil em função de níveis de sombreamento. Horticultura Brasileira, v.25, p.210-204, 2007.

SAEBO, A.; MORTENSEN, L.M. The influence of elevated CO2 concentration on growth of seven grasses and one clover species in a cool maritime climate. Acta Agriculturae Scandinavia Section B-Soil and Plant Science, v.46, p.49-54, 1996.

SANTOS, H.G. dos; JACOMINE, P.K.T.; ANJOS, L.H.C. dos; OLIVEIRA, V.A. de; OLIVEIRA, J.B. de; COELHO, M.R.; LUMBRERAS, J.F.; CUNHA, T.J.F. (Ed.). Sistema brasileiro de classificação de solos. 2.ed. Rio de Janeiro: Embrapa Solos, 2006. $306 \mathrm{p}$.

SHAHAK, Y. Photo-selective netting for improved performance of horticultural crops. A review of ornamental and vegetable studies carried out in Israel. Acta Horticulture, n.770, p.161-168, 2008.

SHAHAK, Y.; GUSSAKOVSKY, E.E.; GAL, E.; GAELEVIN, R. Colornets: crop protection and light-quality manipulation in one technology. Acta Horticulturae, v.659, p.143-161, 2004.

TAIZ, L.; ZEIGER, E. Fisiologia vegetal. Porto Alegre: Artmed, 2004. 719p.

TSORMPATSIDIS, E.; HENBEST, R.G.C.; DAVIS, F.J.; BATTEYA, N.H.; HADLEYA, P.; WAGSTAFFE, A. UV irradiance as a major influence on growth, development and secondary products of commercial importance in Lollo Rosso lettuce 'Revolution' grown under polyethylene films. Environmental and Experimental Botany, v.63, p.232-239, 2008.

VAN DEN DOOL, H.; KRATZ, P.D.J.A. Generalization of the retention index system including linear temperature programmed gas-liquid partition chromatography. Journal of Chromatography, v.11, p.463-471, 1963.

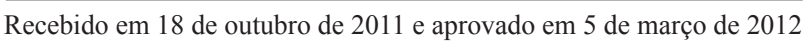

